\title{
A Review of Antiviral Polymer Nano Composites (PNC) in Biomedical Applications; Coronavirus Pandemic Situation
}

\author{
Jawad $\mathrm{AJ}^{1,2 *}$ \\ ${ }^{1}$ University of Babylon, Department of Polymer and Petrochemicals Industrials Engineering, \\ Iraq \\ ${ }^{2}$ Queen Mary University of London, School of Engineering and Materials Science, UK
}

*Corresponding author: Akram Jassim Jawad, University of Babylon, College of Materials Engineering, Department of Polymer and Petrochemicals Industrials Engineering, Queen Mary University of London, School of Engineering and Materials Science, London, UK, Email: akrammaterials4@gmail.com

\section{Review Article}

Volume 5 Issue 1

Received Date: January 08, 2021

Published Date: January 22, 2021

DOI: $10.23880 /$ eij-16000176

\section{Abstract}

Since December 2019, scientists and engineers have tried to minimize and control Covid-19 spreading by different ways. These ways usually focused on cutting the circlet of Covid-19 infection by both direct and non-direct ways. As a polymer composites widely used in every day products, a short review of Polymer Nano Composites (PNCs) for antiviral activity against Covid-19 has been achieved. Firstly, virus's structure, especially Covid-19 has been discussed. Then, different PNCs applications in biomedical that work against coronavirus have been presented and discussed. Also, comprehensive review of antiviral activity mechanisms have been reported with selected examples of PNCs and applications.

Keywords: Biomaterials; Covid-19; Nano Composites; Polymers; Antiviral; Health Care

Abbreviations: PNCs: Polymer Nano Composites; PPE: Personal Protection Equipment; PES: Polyethersulfone; PMMA: Polymethylmethacrylate; PP: Polypropylene; ACE2: Angiotensin-Converting Enzyme 2; ROS: Reactive Oxygen Species; PDMS: Polydimethylsiloxane; PEI: Polyethylenimine

\section{Introduction}

In recent years, Polymer Nano Composites (PNC) field have been developed for many biomedical applications as antiviral, antibacterial, and antifungal agents, and have been used in many products in our daily life [1]. Additionally, the antiviral activity of PNC has reached in the top of the list research of polymer, nanotechnology, and materials science since Covid-19 was discovered in December 2019 [2,3]. Moreover, there were more than 76 million confirmed cases with more than 1.6 million deaths because Covid-19 until Friday 20 December 2020, as it is clear on Johns Hopkins University website, Johns Hopkins University, 2020 [4].
This has high impact on all life sectors, such as economic, education, and social. As PNCs have been used widely in every day products in health care system, for example the personal protection equipment (PPE), the improvement in antiviral activity of these composites could be helpful for both heath system and public society against Covid-19. For example, PNCs based on copper oxide nano particles have high ability to work as an antiviral agent in PPE [5]. In this review, the probable role of PNC as an antiviral agent against coronavirus has been highlighted in terms of the more contact products in human life, such as drug, coating, cloths and house products.

\section{Polymer Nano Composite (PNC) against Coronavirus}

PNC has high opportunity to work against coronavirus because of its scope activity that located in nanoscale measurements, as it shown in Figure 1A [6]. Also, the 
ingredients and structure of coronavirus belong to nano dimensions, which encourage PNC to be a highly recommended factor in this case, as it is clear also from Figure 1B [7]. Generally, the coronavirus spreads by different ways, without contact or with contact as directly or indirectly, as it is clear in Figure 2 [8]. For carrier drops of viruses that less than 5 microns, can spread during the air from infected to non-infected person. In this case, there are two main ways to stopping this spreading, by making social distance or wearing a mask, which mainly could improve it by PNC layer. For carrier drops of viruses that more than 5 microns, can collect on the product surfaces to spread the virus indirectly in a contact situation, or on human body parts such as hands to spread the virus directly in contact case too. To minimize the spreading in these situations, especially in indirectly, in contact case, the products have to be coated with coating based on PNC.
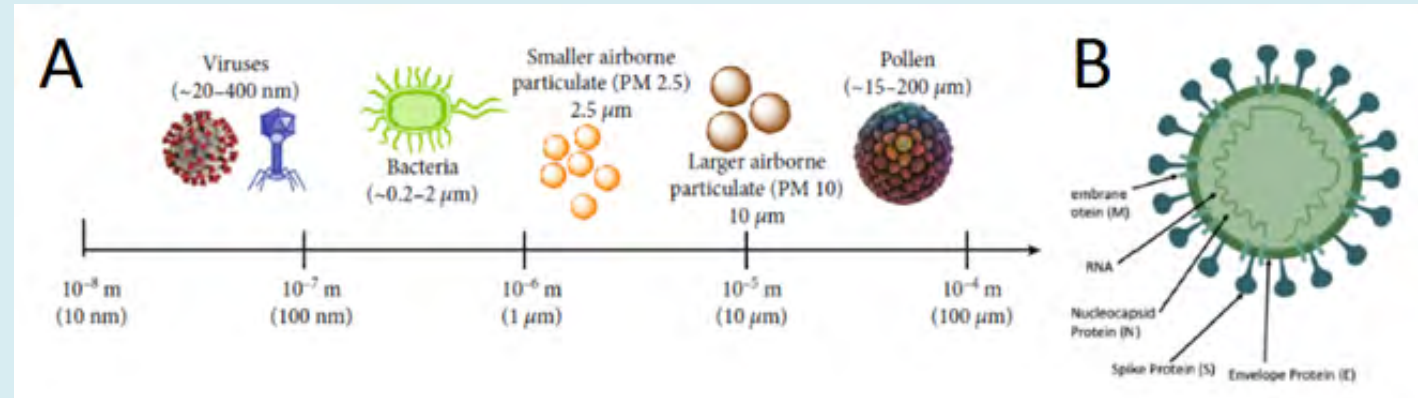

Figure 1: (A) Relative size chart of common airborne contaminants and pathogens [6], (B) Schematic of the main structure of coronavirus [7].

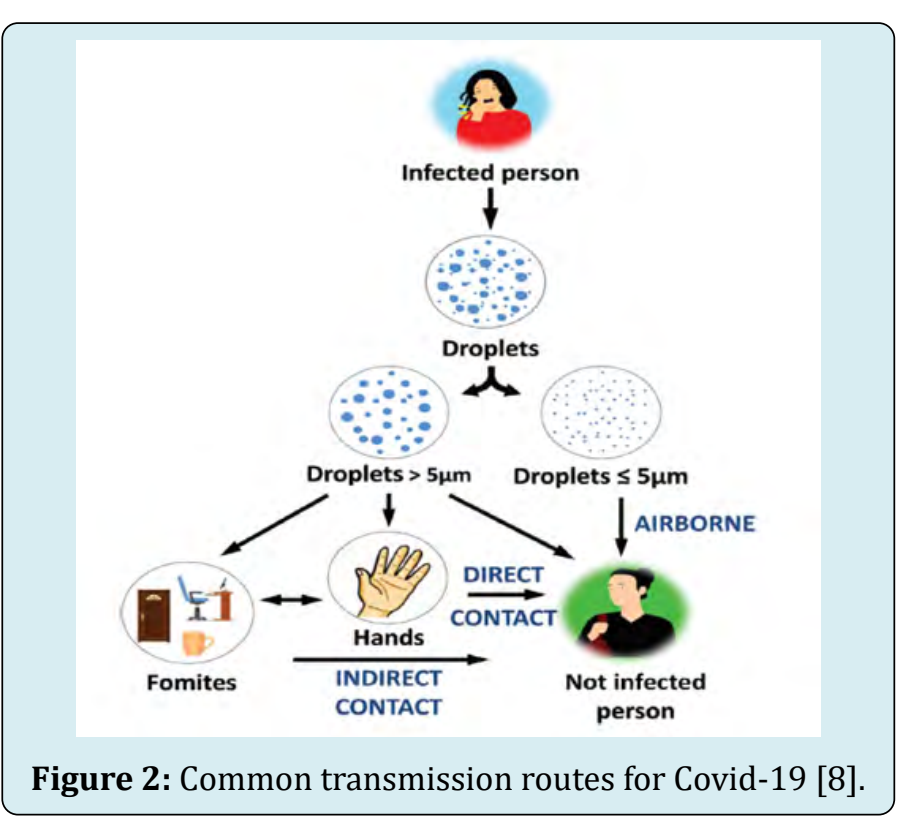

In addition to that, there are different ways and applications of PNC to protect human against viruses, such as drug carrier, drug delivery system, nanoparticles, biopolymers and protective equipment's, as it shown in Figure 3 [8]. Figure 4 represent materials kinds and nano materials based, respectively, which are used as antiviral agents [9]. As PNC have wide important properties such as high mass and easy production, cheap, high ability of sterilization and disinfection comparing with others kinds of materials, they have been used in many biomedical applications, as it was shown in Figure 5 [5]. The antiviral PNC have been prepared, produced and investigated with different filled nanoparticles such as silver, titanium dioxide, and zinc oxide nanoparticles for both human cells, and life products coating applications $[1,21]$. This activity works mainly on the principles of making the barrier between the viruses host cells and other cells of the body or repelling the virus host drops with high repulsive forces on the products and surfaces. These forces have been confirmed to play a significant factor in different virus's activities in vivo and on the product surfaces, which might raise thoughts to apply that in these days to minimize coronavirus infections, and for better management of economic and social life activates [10].

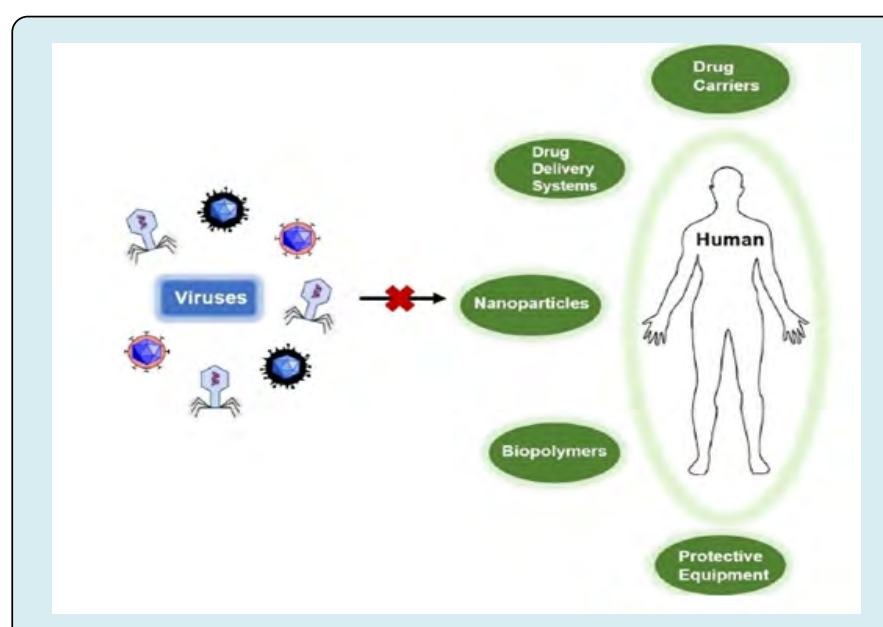

Figure 3: Human protection ways against viruses [8]. 

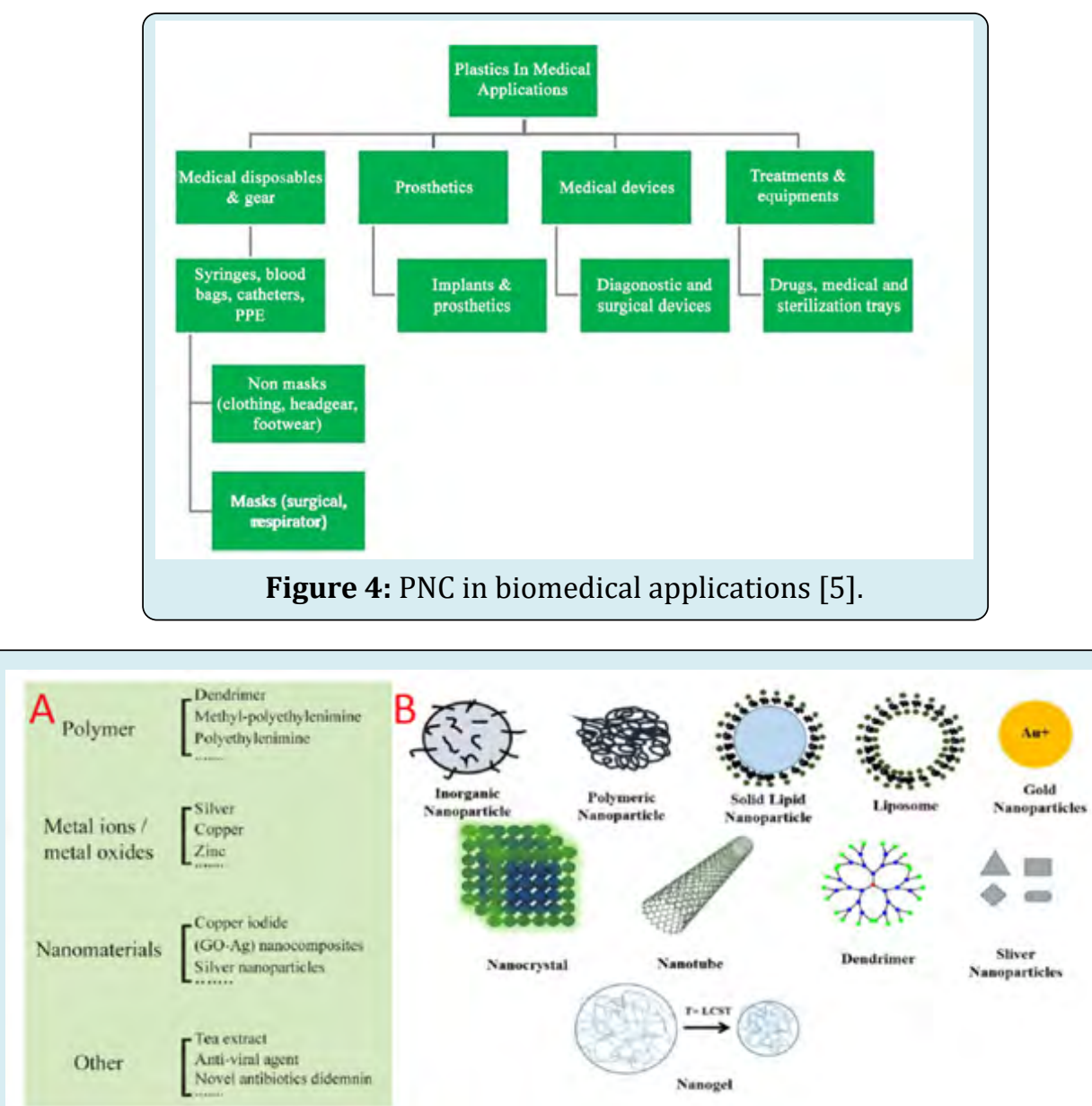

Figure 5: (A) Materials kinds and (B) Nano materials based that used as antiviral agents [9].

An important application also that PNC based on polyethersulfone (PES), polymethylmethacrylate (PMMA), and polypropylene (PP) have been used in plasmapheresis process to collect blood plasma from recovered patients who infected by Covid-19 and gives it to another infected patients [5]. Another important application is using PNC as an antiviral agent such as antiviral coating, antiviral drug and medical devices like personal protective equipment (PPE) that could help in coronavirus pandemic situation, where the necessary time to kill the viruses has been found within ten minutes of virus exposure in average [5].
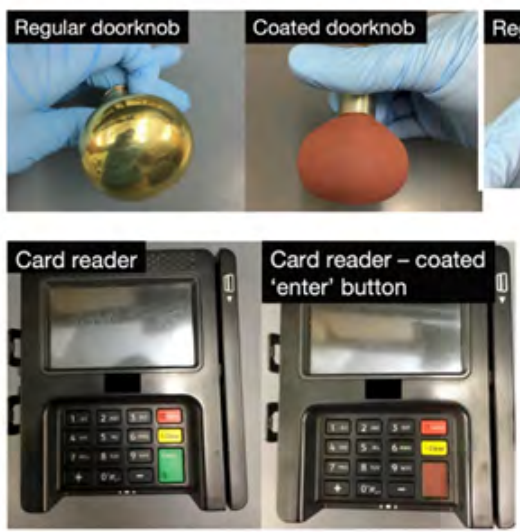
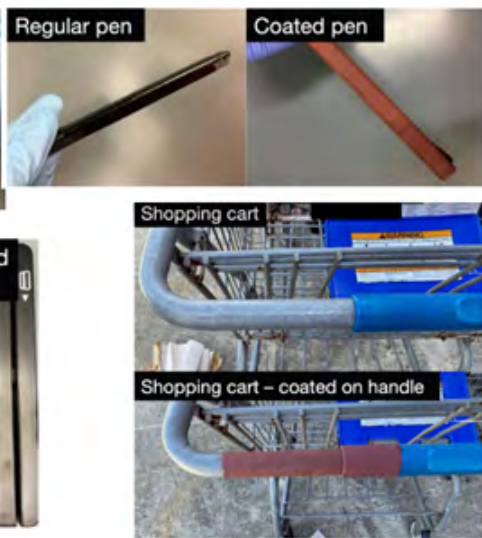

Figure 6: Some products that were coated by Cu20/polyurethane as a PNC for its antiviral activity against coronavirus [11]. 


\section{Epidemiology International Journal}

Figure 6 illustrates some products that were coated by Cu20/polyurethane as a PNC for its antiviral activity against coronavirus [11]. Figure 7 summarizes using PNC for therapy of Covid-19 by different ways [12]. The First approach may be done by adding nano additives into PPE to prevent catching virus by the respiratory system. Another way could be by applying nanomaterials into the drug delivery system to the pulmonary system by inhalators. Also, using PNC to preventing cellular binding of virus by targeting angiotensinconverting enzyme $2\left(\mathrm{ACE}_{2}\right)$ receptors or viral S protein in virus membrane.

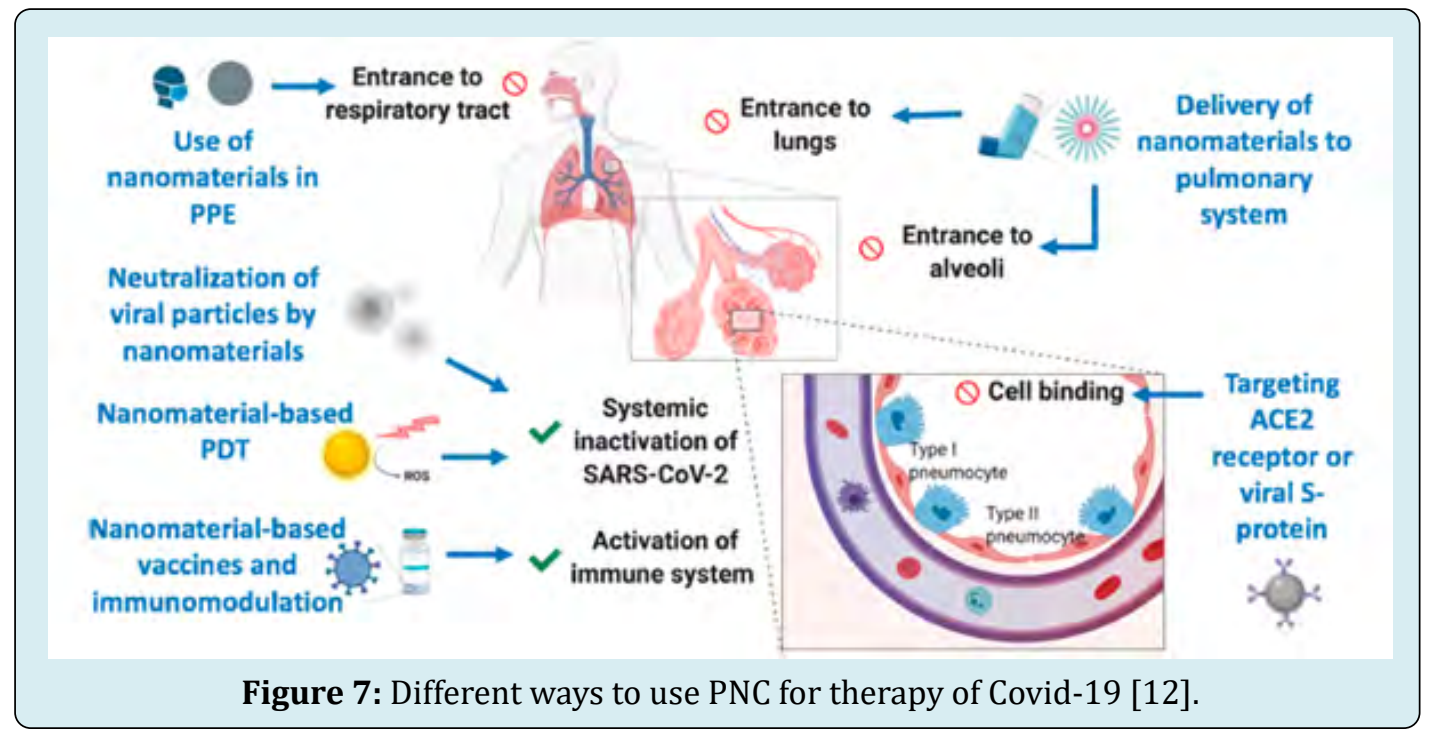

\section{Mechanisms of Antiviral}

There are three mechanisms of antiviral activities for artificial materials, which are depending on heat, ions or small molecules techniques, as it is clear in Figure 8 [13]. For example, PNC based on cupper nano particles work as antiviral surfaces by heat generation technique because of its high heat connectivity properties, as it was shown in Figure 9 [14]. Figure 10 illustrates ions technique for an antiviral activity of PNC [8]. In which some PNC materials might produce an ions by light that work as reactive oxygen species (ROS), which damages of the membrane, proteins and DBA/ RNA of viruses. Small molecules such as nano particles or some polymer chains could make a barrier between the host and virus to prevent Covid-19 spreading, as it is clear in Figure 11, which represents an antiviral activity by small molecules (nano) technique [15].

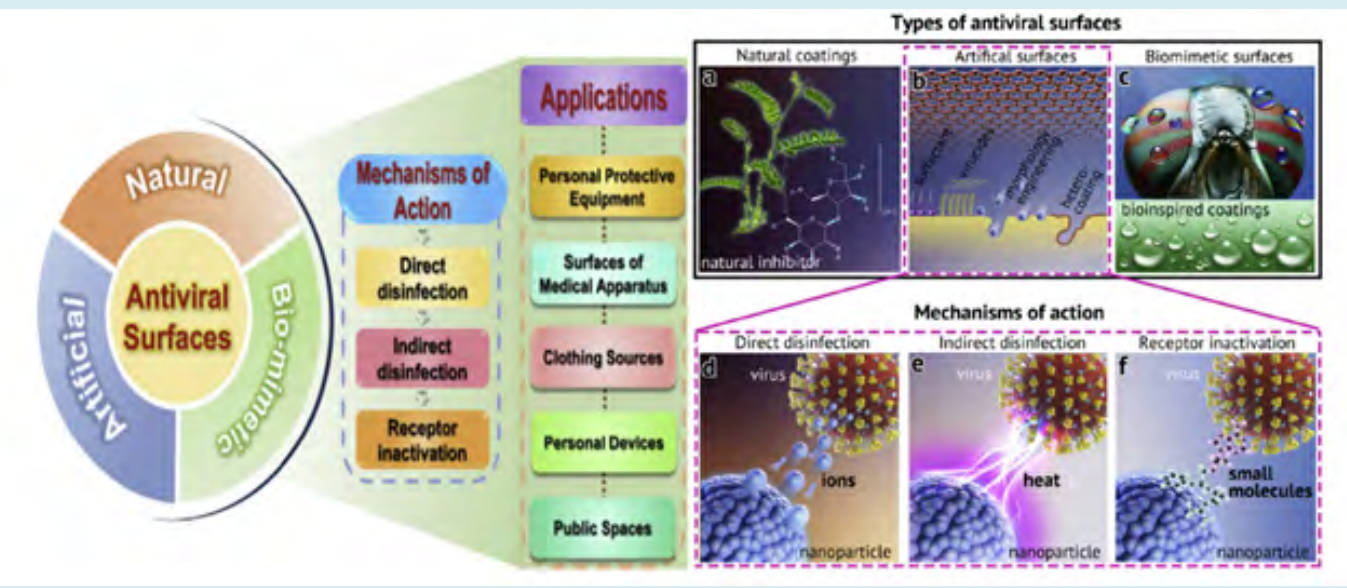

Figure 8: Concept of antiviral surfaces, their action mechanisms, and the potential applications [13]. 


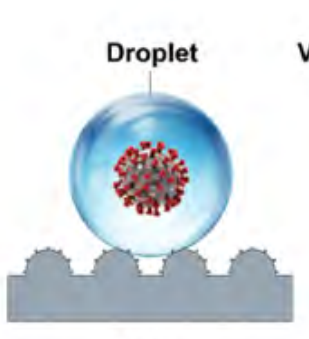

(a)

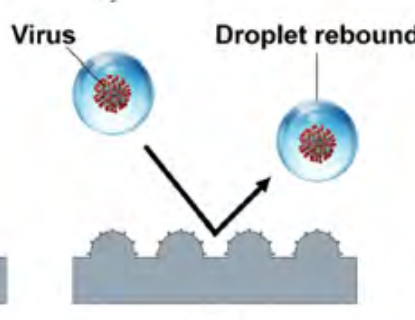

(b)

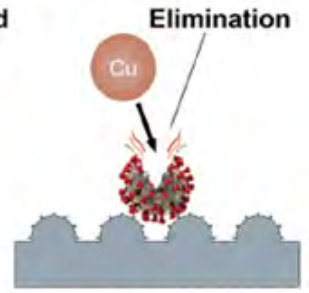

(c)

Figure 9: A schematic illustration of antiviral activity by heat technique of PNC based on Copper nano particles in three different stages: a Virus encapsulation, b contamination suppression, and c virus Elimination [14].

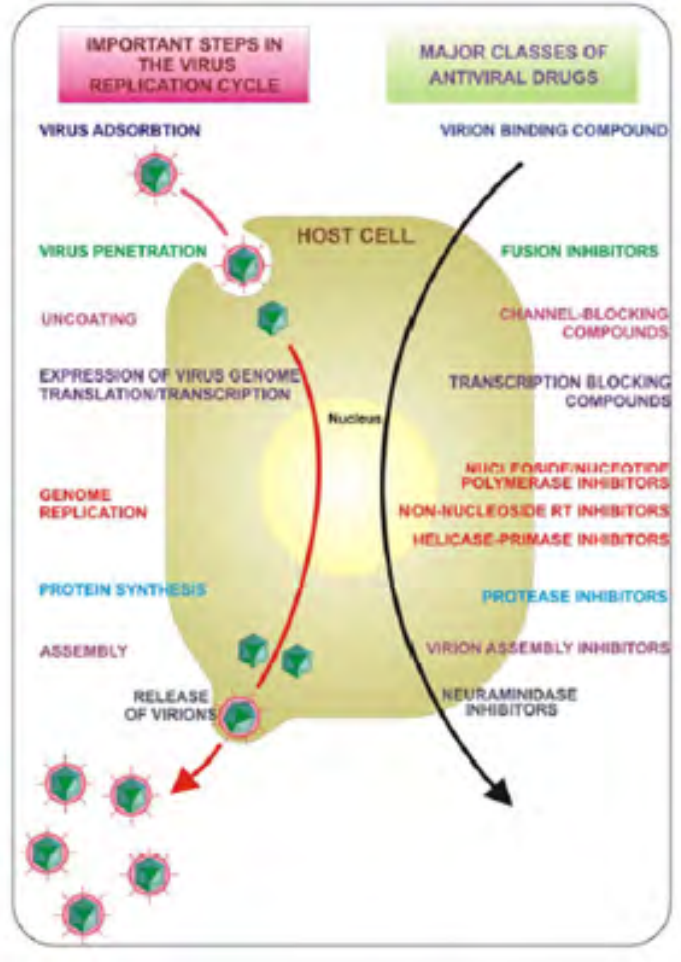

Figure 10: Photodynamic inhibition of viruses by reactive oxygen species (ROS) induced damage of the membrane, proteins and DBA/RNA [8].

There are different ingredients of PNC that work as antiviral agent [16]. For example, PNC coating may consist of pharmaceutically admissible salts of chlorhexidine, and a lubricating agent that does not significantly be adsorbed to the anti-infective agent. Also, some PNC structures have a porous plastic layers and antiviral agents, which could be applied into vents, and filters applications. Another PNC has high hydrophilic or self-cleaning ability to repel viruses away from surfaces. A successful example to use PNC as a coating layer for antiviral applications is polycationic PEI, as it shown in Figure 12 in the schematic representation of its antiviral mechanism against virus [8]. For PNC based on copper nano particles, the antiviral activity might be simply started by adsorption of microorganisms such as coronavirus on the PNC surface, as it was shown in Figure 13 [19]. Then, diffusion of water through the PNC layer to start the corrosion process by oxygen and copper ions. Consequently, copper ions damage the microorganism cell membrane, to damage RNA also.

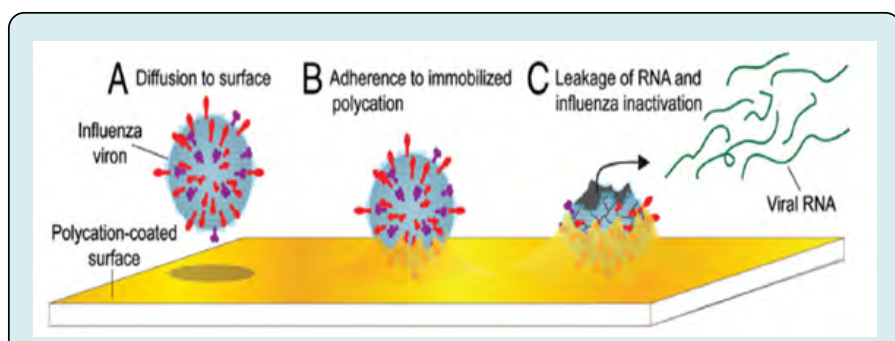

Figure 12: Schematic representation of antiviral mechanism of virus inactivation in polycationic PEI derivative coatings [8].

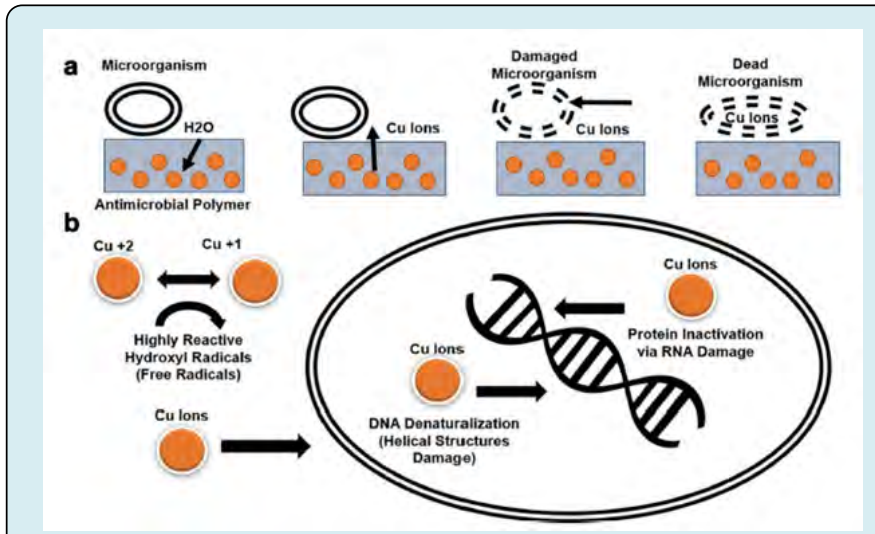

Figure 13: Theoretical mechanisms of PNC based on copper nano particles [17]. 


\section{PNC in Drug System}

One of the most important biomedical applications of PNC is in the drug industries and drug delivery systems especially as an antiviral drug, for example heparin polysaccharides against HPV16 [2]. Martinez LS, et al. [2] studied the antiviral ability of polysaccharides polymers with oligomers of sulfated-glycomimetic in vivo for different kinds of viruses such as HPV16, Influenza a Virus (143 IAV), and Virus (HSV) [2].

As we can see in Figure 14 that illustrates the schematic model proposed of the inhibition mechanism for antiviral PNC by competing with virus to prevent it enters the cells. The results shown that these polymers could prevent spreading the viruses inside the human tissue environment by providing a barrier and covering layers around the active sites on cells wall, which are targeted by viruses [2]. These results might open the light on the possibility of those types of PNC to apply against Covid-19 spreading, at least to manage and control the high critical cases that have infected by a high percentage of coronavirus. Figure 15 shows different forms of carriers based on PNC that work as an antiviral agent [18].

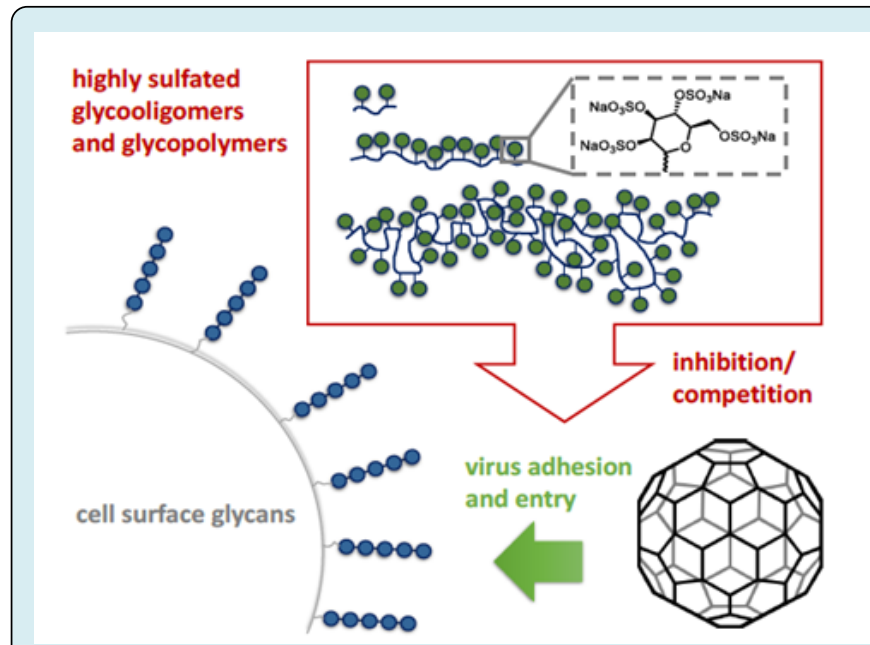

Figure 14: A schematic model represents the inhibition mechanism for antiviral PNC by competing with virus to prevent it enters the cells [2].

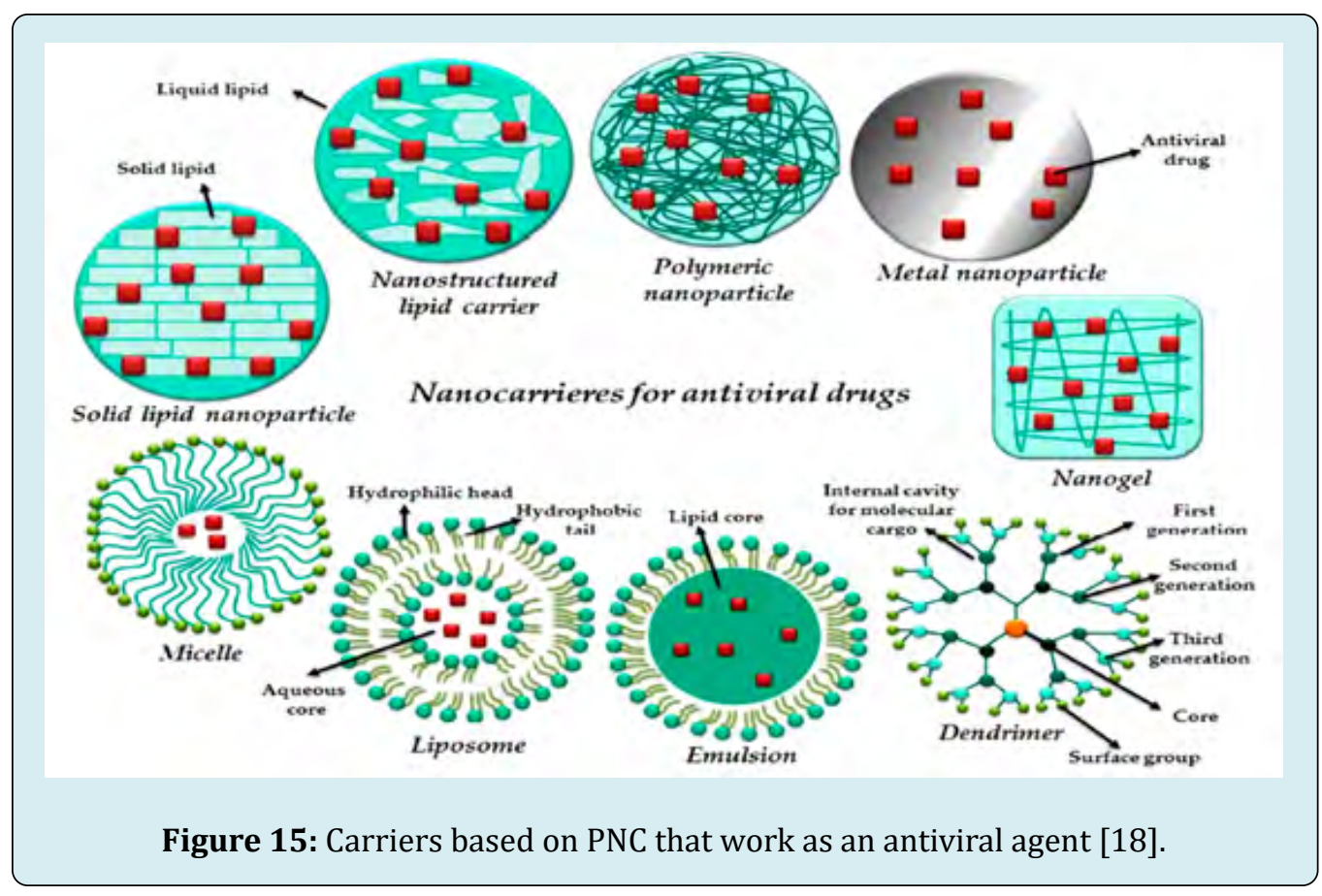

\section{PNC in Daily Products}

PNC has been used in a wide range of daily routine applications especially that are in contact with human directly, such as cloths, and fabric industries, and products. The most popular antiviral nanoparticles loaded in polymer including silver, copper, zinc oxide, titanium dioxide, and many metals oxides [1]. Antiviral based-cotton fabrics have been coated by polydimethylsiloxane (PDMS) which was loaded with Couper oxide $(\mathrm{CuO})$ nanoparticles by using different cross linkers [1]. Figure 16 shows the contact angle of a water drop on cotton fabric with and without the coating layer of PDMS/ CuO. The high contact angle of coated fabric might give a chance to decrease spreading Covid-19, especially for people who work in high environment of viruses, such as hospitals and healthcare centres. 


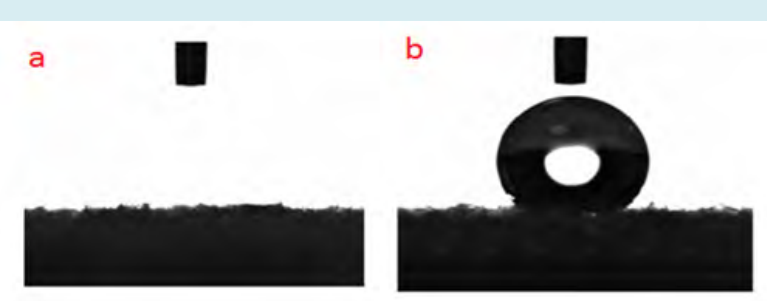

Figure 16: Pictures of the contact angle of a water drop on cotton fabric; a) without, b) with the coating layer of PDMS/CuO [1].

It has been discovered that polycations polymers that has hydrophobic ability and insoluble in water, such as $\mathrm{N}$, $\mathrm{N}$-dodecyl methyl-polyethylenimine (PEI), can kill bacteria and viruses by high polycationic chains forces [10]. These forces destroy the lipid layer membrane that protects the viruses, which might works probably with Covid-19. For example, it can apply to coat the walls, doors, windows and all high contact surfaces to make these surfaces have selfantiviral ability. Another important example of PNC coating that has high ability of antiviral activity was illustrated by Figure 17 [19]. As it has been discovered that The PVA based $\mathrm{Cu}-\mathrm{Gr}$ (copper-graphene) nanocomposite can be used to generate a highly transparent antiviral coating of the cell phone screen [19].

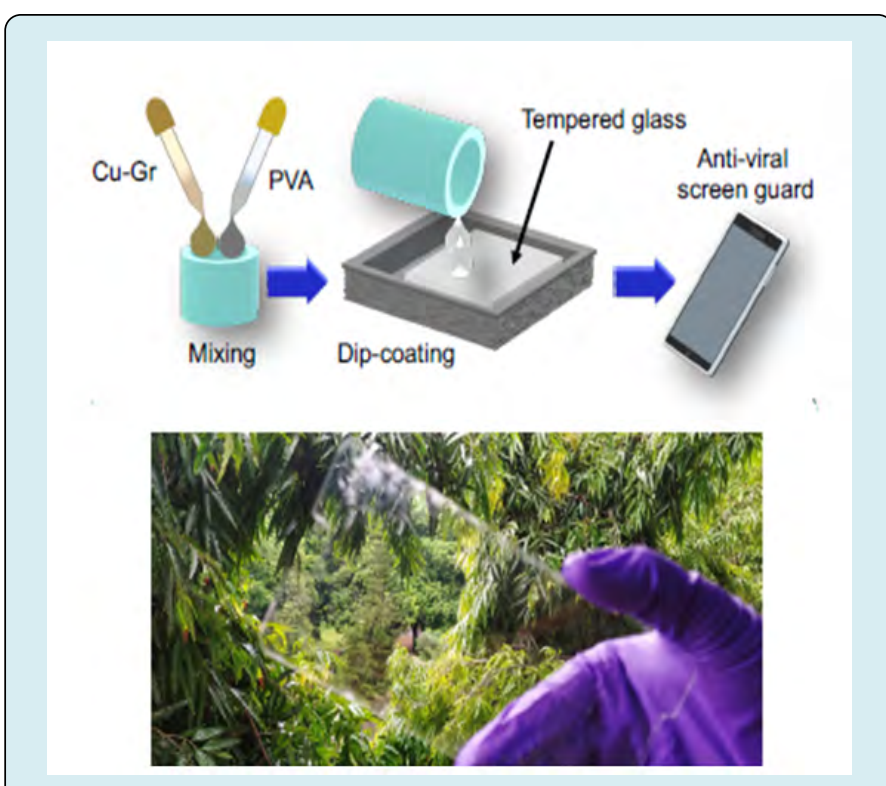

Figure 17: PVA based $\mathrm{Cu}-\mathrm{Gr}$ (copper-graphene) nanocomposite can be used to generate a highly transparent antiviral coating of the cell phone screen [19].

Additionally, one of the important applications of antiviral ability of PNC is to apply its layer in PPE, such as the mask fabrication to increase its ability to non-infectious and preventing viruses spreading. As an example, adding fourth layer to three layers mask structure that shown in Figure 18, to produce four layers mask structure that shown in Figure $19[6,20,21]$. An example of PNC that could use is based on nylon 6/poly acrylonitrile or polyethylene/polypropylene nanofibers layer that have been prepared by electrospinningnetting technique, as it were shown in Figure 20 [6].

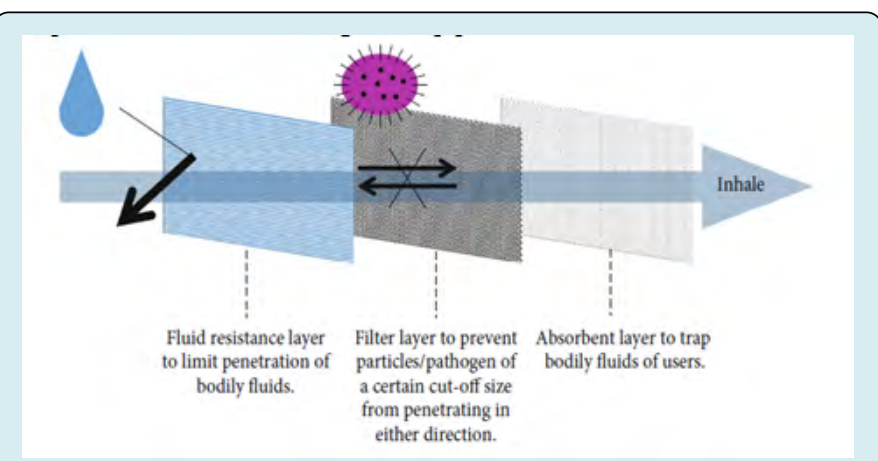

Figure 18: Illustration showing three layers mask structure [6].
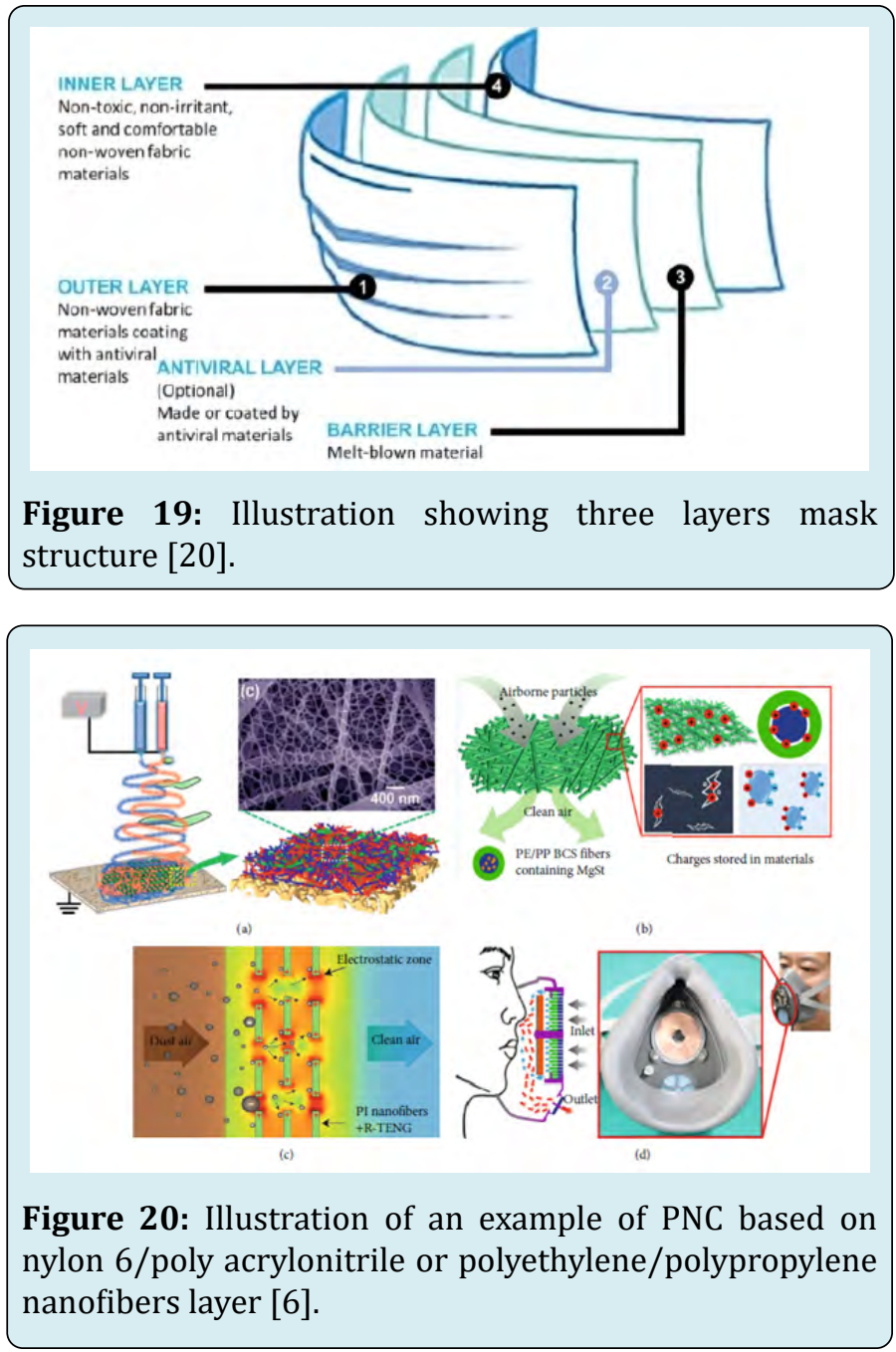


\section{Conclusion}

Polymer Nano Composites (PNC) could be a significant factor in the developing process of antiviral drug toward Covid-19, such as polysaccharides nanocomposites. Another PNC might play a crucial solder in the cutting of coronavirus circle spreading's by introduce a barrier between productproduct and product-human infections. This can be a simple and limited products like spoons and door knobs, or even wide range application uses such as entirely wall coating of house walls. Consequently, PNC could save spending the money and energy, management the health system, decreasing the lockdown periods and social distancing as well.

\section{References}

1. Agrawal N, Low PS, Tan JSJ, Fong EWM, Lai Y, et al. (2020) Durable easy-cleaning and antibacterial cotton fabrics using fluorine-free silane coupling agents and $\mathrm{CuO}$ nanoparticles. Nano Materials Science 2(3): 281-291.

2. Martinez LS, Bauer S, Giesler M, Schelhaas S, Materlik J, et al. (2020) Prophylactic Antiviral Activity of Sulfated Glycomimetic Oligomers and Polymers. J Am Chem Soc 142(11): 5252-5265.

3. Jawad AJ (2020) Effectiveness of population density as natural social distancing in COVID19 spreading. Ethics Med Public Health 15: 100556.

4. (2020) Johns Hopkins University coronavirus resource center.

5. Gadhave RV, Vineeth SK, Gadekar PT (2020) Polymers and Polymeric Materials in COVID-19 Pandemic: A Review. Open Journal of Polymer Chemistry 10(3): 6675.

6. Chua MH, Cheng W, Goh SS, Kong J, Li B, et al. (2020) Face masks in the new COVID-19 normal: Materials, testing, and perspectives. Research (Wash DC).

7. Palmieri V, Papi M (2020) Can graphene take part in the fight against COVID-19. Nano Today 33: 100883.

8. Hitzky ER, Darder M, Wicklein B, Garcia CR, Sampedro RM, et al. (2020) Nanotechnology Responses to COVID-19. Advanced Healthcare Materials 9(19): 2000979.

9. Gurunathan S, Qasim M, Choi Y, Do JT, Park C, et al. (2020) Antiviral potential of nanoparticles-Can nanoparticles fight against coronaviruses? Nanomaterials 10(9): 1645.

10. Haldar J, An D, de Cienfuegos LA, Chen J, Klibanov AM (2006) Polymeric coatings that inactivate both influenza virus and pathogenic bacteria. Proc Natl Acad Sci USA 103(47): 17667-17671.

\section{Epidemiology International Journal}

11. Behzadinasab S, Chin A, Hosseini M, Poon L, Ducker WA (2020) A surface coating that rapidly inactivates SARSCoV-2. ACS Appl Mater Interfaces 12(31): 34723-34727.

12. Weiss C, Carriere M, Fusco L, Capua I, Regla Nava JA, et al. (2020) Toward Nanotechnology-Enabled Approaches against the COVID-19 Pandemic. ACS Nano 14(6): 63836406.

13. Sun Z, Ostrikov K (2020) Future antiviral surfaces: Lessons from COVID-19 pandemic. Sustainable Materials and Technologies 25: 00203.

14. Meguid SA, Elzaabalawy A (2020) Potential of combating transmission of COVID-19 using novel self-cleaning superhydrophobic surfaces: part I-protection strategies against fomites. International Journal of Mechanics and Materials in Design 16(3): 423-431.

15. Galdiero S, Falanga A, Vitiello M, Cantisani M, Marra V, et al. (2011) Silver nanoparticles as potential antiviral agents. Molecules 16(10): 8894-8918.

16. Pemmada R, Zhu X, Dash M, Zhou Y, Ramakrishna S, et al. (2020) Science-Based Strategies of Antiviral Coatings with Viricidal Properties for the COVID-19 Like Pandemics. Materials 13(18): 4041.

17. Zuniga JM, Cortes A (2020) The role of additive manufacturing and antimicrobial polymers in the COVID-19 pandemic. Expert Rev Med Devices 17(6): 477-481.

18. Cojocaru FD, Botezat D, Gardikiotis I, Uritu CM, Dodi G, et al. (2020) Nanomaterials designed for antiviral drug delivery transport across biological barriers. Pharmaceutics 12(2): 171.

19. Jana ID, Kumbhakar P, Banerjee S, Gowda CC, Kedia $\mathrm{N}$, et al. (2020) Development of a copper-graphene nanocomposite based transparent coating with antiviral activity against influenza virus. BioRxiv.

20. Liang L, Ahamed A, Ge L, Fu X, Lisak G (2020) Advances in Antiviral Material Development. ChemPlusChem 85(9): 2105-2128.

21. Jawad AJ, Jassim AE, Hadi NJ (2020) Effect of Titanium Dioxide Nanoparticles $\left(\mathrm{TiO}_{2}\right.$ NPs $)$ on Rheological Characteristics Behavior of Poly Vinyl Acetate (PVAc). NanoWorld J 6(3): 61-65. 